\title{
Superior nationwide outcomes of endovascular versus open repair for isolated descending thoracic aortic aneurysm in 11,669 patients
}

\author{
Raja R. Gopaldas, MD, ${ }^{\text {a,b }}$ Joseph Huh, MD, ${ }^{\text {a,b }}$ Tam K. Dao, PhD, ${ }^{\text {c }}$ Scott A. LeMaire, MD, ${ }^{\text {a,b }}$ \\ Danny Chu, MD, ${ }^{\mathrm{a}, \mathrm{d}}$ Faisal G. Bakaeen, MD, ${ }^{\mathrm{a}, \mathrm{d}}$ and Joseph S. Coselli, MD ${ }^{\mathrm{a}, \mathrm{b}}$
}

Objectives: Thoracic endovascular aneurysm repair (TEVAR) was introduced in 2005 to treat descending thoracic aortic aneurysms. Little is known about TEVAR's nationwide effect on patient outcomes. We evaluated nationwide data regarding the short-term outcomes of TEVAR and open aortic repair (OAR) procedures performed in the United States during a 2-year period.

Methods: From the Nationwide Inpatient Sample data, we identified patients who had undergone surgery for an isolated descending thoracic aortic aneurysm from 2006 to 2007. Patients with aneurysm rupture, aortic dissection, vasculitis, connective tissue disorders, or concomitant aneurysms in other aortic segments were excluded. Of the remaining 11,669 patients, 9106 had undergone conventional OAR and 2563 had undergone TEVAR. Hierarchic regression analysis was used to assess the effect of TEVAR versus OAR after adjusting for confounding factors. The primary outcomes were mortality and the hospital length of stay (LOS). The secondary outcomes were the discharge status, morbidity, and hospital charges.

Results: The patients who had undergone TEVAR were older $(69.5 \pm 12.7$ vs $60.2 \pm 14.2$ years; $P<.001)$ and had higher Deyo comorbidity scores $(4.6 \pm 1.8$ vs $3.3 \pm 1.8 ; P<.001)$. The unadjusted LOS was shorter for the TEVAR patients ( $7.7 \pm 11$ vs $8.8 \pm 7.9$ days), but the unadjusted mortality was similar (TEVAR $2.3 \%$ vs OAR $2.3 \% ; P=1.0$ ). The proportion of nonelective interventions was similar between the 2 groups (TEVAR $15.9 \%$ vs OAR $15.8 \% ; P=.9)$. The TEVAR and OAR techniques produced similar risk-adjusted mortality rates; however, the TEVAR patients had $60 \%$ fewer complications overall (odds ratio, $0.39 ; P<.001$ ) and a shorter LOS (by 1.3 days). The TEVAR patients' hospital charges were greater by $\$ 6713$ (95\% confidence interval $\$ 1869$ to $\$ 11,556 ; P<.001)$. However, the TEVAR patients were 4 times more likely to have a routine discharge to home.

Conclusions: The nationwide data on TEVAR for descending thoracic aortic aneurysms have associated this procedure with better in-hospital outcomes than OAR, even though TEVAR was selectively performed in patients who were almost 1 decade older than the OAR patients. Compared with OAR, TEVAR was associated with a shorter hospital LOS and fewer complications but significantly greater hospital charges. (J Thorac Cardiovasc Surg 2010;140:1001-10)

The first successful open repair of a thoracic aortic aneurysm with a prosthetic graft was reported in 1953 by DeBakey and Cooley, ${ }^{1}$ and this procedure has stood the test of time. However, the introduction of endovascular interven-

\footnotetext{
From the Division of Cardiothoracic Surgery, ${ }^{\mathrm{a}}$ Michael E. DeBakey Department of Surgery, Baylor College of Medicine; The Texas Heart Institute at St. Luke's Episcopal Hospital $^{\text {b; }}$ the Department of Educational Psychology, ${ }^{\mathrm{c}}$ University of Houston; and the Michael E. DeBakey Veterans Affairs Medical Center, ${ }^{\mathrm{d}}$ Houston, Tex.

Disclosures: Dr Coselli reports relationships with Cook Inc.: PI on the TX2 Thoracic Stent Graft Trial; Medtronic, Inc.: PI on the Valor II and THRIVE Stent Graft Trial, consultant in 2009, and speaker in 2009; and WL Gore \& Associates, Inc.: PI on the Gore Conformable Descending/Dissection Thoracic Stent Graft Trial, consultant in 2010, and speaker in 2010.

Read at the 90th Annual Meeting of The American Association for Thoracic Surgery, Toronto, Ontario, Canada, May 1-5, 2010.

Dr Gopaldas is with the Division of Cardiothoracic Surgery, University of MissouriColumbia, Columbia, Mo.

Received for publication April 29, 2010; revisions received Aug 3, 2010; accepted for publication Aug 10, 2010.

Address for reprints: Raja R. Gopaldas, MD, Division of Cardiothoracic Surgery, University of Missouri-Columbia, One Hospital Dr., MA312, Columbia, MO 65212 (E-mail: gopaldasr@health.missouri.edu).

$0022-5223 / \$ 36.00$

Copyright (C) 2010 by The American Association for Thoracic Surgery

doi:10.1016/j.jtcvs.2010.08.007
}

tions for the management of abdominal aortic aneurysm repair in 1991 caused a major paradigm shift, and the latter technique has become the primary surgical modality for a significant number of patients with an isolated abdominal aortic aneurysm. ${ }^{2-4}$ In 2005 , consequent to the results of the Phase II trial of the GORE TAG endovascular prosthesis (WL Gore \& Associates, Newark, Del), the technology for thoracic endovascular aneurysm repair (TEVAR) was approved in the United States for repair of descending thoracic aortic aneurysms (DTAAs). ${ }^{5}$ Since then, the use of stent grafts has increased and has expanded to include hybrid approaches for treating certain complex thoracic and thoracoabdominal aneurysms, although many of these applications are off label. ${ }^{6}$

Several single-center reports have described the intermediate-term outcomes of TEVAR. ${ }^{7,8}$ However, these data have not provided insight into the nationwide utility or effectiveness of the procedure. Typically, the success of any surgical procedure will be dictated by its outcomes and ease of use. Procedures that are easy to perform are likely to be adopted by-and performed in-many 


\author{
Abbreviations and Acronyms \\ CI = confidence interval \\ DTAA $=$ descending thoracic aortic aneurysm \\ FDA $=$ Food and Drug Administration \\ HCUP $=$ Healthcare Cost and Utilization Project \\ ICD-9- = International Classification of \\ CM Diseases, Ninth Revision, Clinical \\ Modification \\ LOS = length of stay \\ NIS $=$ US Nationwide Inpatient Sample \\ OAR = open aortic repair \\ TEVAR $=$ thoracic endovascular aneurysm repair
}

centers. In contrast, technically challenging procedures are likely to be restricted to a few high-volume centers. ${ }^{9}$ Thus far, little is known about TEVAR's nationwide use and effect on patient outcomes. Therefore, we compared the inhospital outcomes of TEVAR and open DTAA procedures performed in the United States during the initial 2-year period after Food and Drug Administration (FDA) approval.

\section{MATERIALS AND METHODS Data Source}

Data were obtained from the US Nationwide Inpatient Sample (NIS), a database of hospital inpatient stays maintained by the Agency for Healthcare Research and Quality as a part of the Healthcare Cost and Utilization Project (HCUP).${ }^{10}$ We used only part of the post-FDA-approval data that were available; these data had been collected in 2006 and 2007. The NIS is the largest all-payer inpatient care database, representing $90 \%$ of all hospital discharges from nonfederal facilities in the United States and, thus, was particularly suited for a nationwide comparison. The NIS has numerous internal quality assurance procedures for checking the consistency and validity of data (available from: www.hcup-us.ahrq.gov/db/quality.jsp). Furthermore, the HCUP validates the NIS annually by comparing its contents with those of 2 similar databases, the National Hospital Discharge Survey and the Medicare Provider Analysis and Review, to assess potential biases in the data set (available from: www.hcup-us.ahrq.gov/db/nation/nis/nisrelated reports.jsp). The NIS contains data on approximately 8 million hospital stays annually from more than 1000 hospitals, although this number has varied slightly on an annual basis. Weights based on sampling probabilities for each stratum are used in the analysis to ensure that the hospitals studied are representative of all US hospitals.

The variables available in the NIS database include patient and hospital demographics, payer information, treating and concomitant diagnoses, inpatient procedures, in-hospital mortality, and the hospital length of stay (LOS) (the date of admission to the date of discharge). The NIS database also captures up to 15 International Classification of Diseases, Ninth Revision, Clinical Modification (ICD-9-CM) diagnostic and procedure codes per admission.

The institutional review board of Baylor College of Medicine approved the present study, which was deemed exempt from the informed consent requirement because of the nonidentifiable nature of the data. The reported data conformed to the data-use agreement for the NIS from the HCUP. Additional information about the NIS is available from the Agency for Healthcare Research and Quality (available from: www.hcup-us.ahrq. gov/nisoverview.jsp).

\section{Patient Selection}

From the weighted NIS data for the calendar years 2006 and 2007, we used the ICD-9-CM diagnosis and procedure codes (see Appendix 1) ${ }^{11}$ to identify all patients aged 18 years or older who had had a diagnosis of DTAA and had undergone open aortic repair (OAR) or TEVAR. Patients with vasculitis, any connective tissue disorder, gonadal dysgenesis, aneurysmal rupture, aortic dissection, or concomitant aneurysms in other aortic segments were excluded, as were patients who had undergone both OAR and TEVAR (because the database had no mechanism to indicate whether these were intraoperative conversions or planned staged procedures). Because our study was retrospective, our analysis compared the outcomes of the actual procedures, rather than the intent-to-treat.

The Deyo index was used to compare the 2 groups in terms of preoperative morbidity. ${ }^{12}$ The Deyo index-a weighted comorbidity index modified from the Charlson comorbidity index-assesses 17 prespecified comorbidities and is specifically designed to be used with administrative databases, using more than 600 ICD-9-CM diagnosis codes to query specific comorbid diagnoses. In previous surgical studies, we and other investigators have demonstrated the feasibility of querying the NIS database for cardiac surgical procedures. ${ }^{13,14}$ Up to 15 ICD-9-CM diagnosis codes were recorded per patient. These codes were then used to produce a summary Deyo index score for each patient. The Deyo index and its individual components were used for risk adjustment in the statistical model.

\section{Study End Points}

The primary outcomes of interest for the present study were in-hospital mortality, defined as death during the index hospitalization, and LOS. The secondary end points were discharge status, in-hospital morbidity, and hospital charges. Discharges were classified as either routine or nonroutine. A routine discharge was defined as discharge home without any sort of home health care, including home intravenous medication therapy. Nonroutine discharges included discharge to a skilled nursing or intermediate care facility or to home with home health care, as well as departure from the hospital against medical advice.

All-cause in-hospital morbidity was recorded according to the ICD-9CM diagnosis codes. The following morbidity categories were assessed: intraoperative/procedure-related complications, postoperative infections, neurologic complications, renal complications, respiratory complications, deep vein thrombosis, and pulmonary embolism. Intraoperative and procedure-related complications were specifically defined as complications that occurred during a procedure or that were directly related to technical aspects of the procedure (see Appendix 2).

\section{Statistical Analysis}

Because of the large size of the NIS data sets, the Statistical Package for Social Sciences, version 17.0 (SPSS, Inc, Chicago, Ill) complex module was used for all statistical analyses. The Student $t$ test and $\chi^{2}$ statistics were computed to examine intergroup differences in age, race, gender, patient income (by quartile, as encoded in the NIS databases), expected payer, Deyo index score, mortality, hospital LOS, and discharge status. After univariate analysis, multivariable analysis with hierarchic linear and logistic regression methods was performed. Hierarchic logistic regression analysis was used to examine the risk-adjusted association of the type of surgical intervention with the in-hospital complications, mortality, and discharge status. In-hospital complications were analyzed both as a whole and in separate categories. Hierarchic linear regression models were used to test riskadjusted associations between the type of operation and the number of complications per admission, LOS, and hospital charges. Because of the large sample size, we knew that our analyses could reveal differences that were statistically significant but of minimal clinical significance. Therefore, 3 different effect size statistics were computed to assess the practical implications of our findings. Cohen's $d$ was calculated for continuous data by using pooled standard deviations and was appropriately weighted for unequal sample size. ${ }^{15}$ The phi $(\varphi)$ coefficient was computed 
TABLE 1. Patient demographics and baseline characteristics

\begin{tabular}{|c|c|c|c|c|c|}
\hline Characteristic & OAR $(n=9106)$ & TEVAR $(n=2563)$ & $\chi^{2}$ or $t$ & $P$ value & Effect size* \\
\hline Age (y) & $60.2 \pm 14.2$ & $69.5 \pm 12.7$ & 31.888 & $<.001$ & 0.67 \\
\hline Elective admission & $7660(84.1 \%)$ & $2158(84.2 \%)$ & 0.009 & .9 & NA \\
\hline Female & $2918(32.0 \%)$ & $1041(40.6 \%)$ & 65.554 & $<.001$ & 0.075 \\
\hline Primary expected payer $\dagger$ & & & 539.804 & $<.001$ & 0.215 \\
\hline Medicare & $3861(42.4 \%)$ & $1716(67.0 \%)$ & & & \\
\hline Private insurance & $4490(49.4 \%)$ & $641(25.0 \%)$ & & & \\
\hline Income quartile $\ddagger$ & & & 216.621 & $<.001$ & 0.138 \\
\hline First & $1553(17.6 \%)$ & $683(27.4 \%)$ & & & \\
\hline Second & $1962(22.2 \%)$ & $685(27.4 \%)$ & & & \\
\hline Third & $2330(26.4 \%)$ & $592(23.7 \%)$ & & & \\
\hline Fourth & $2984(33.8 \%)$ & $537(21.5 \%)$ & & & \\
\hline Hospital size (large) & $7184(78.9 \%)$ & $1869(72.9 \%)$ & 132.385 & $<.001$ & 0.107 \\
\hline Race $\oint$ & & & 163.174 & $<.001$ & 0.141 \\
\hline White & $5656(88.7 \%)$ & $1446(78.2 \%)$ & & & \\
\hline Black & $301(4.7 \%)$ & $190(10.3 \%)$ & & & \\
\hline Deyo comorbidity score & $3.3 \pm 1.8$ & $4.6 \pm 1.8$ & 31.75 & $<.001$ & 0.72 \\
\hline
\end{tabular}

For categorical variables, data are presented as number ( $\%)$, and $\chi^{2}$ test was used; for continuous variables, data are presented as mean \pm standard deviation, and $t$ test was used. $O A R$, Open aortic repair; TEVAR, thoracic endovascular aneurysm repair. *Cohen's d was used for age and Deyo score, and phi $(\varphi)$ was used for female gender. Cramer's V was computed for all other variables. †Valid data were available for 11,659 patients $(99.9 \%)$. $\ddagger$ Valid data were available for 11,327 patients $(97.1 \%)$. $\S$ Valid data were available for 8224 patients $(70.5 \%)$.

for $\chi^{2}$ tests for independence with 1 degree of freedom. Cramer's V was computed for variables with more than 1 degree of freedom. ${ }^{16}$ The following cutoff values were used to judge the computed effect sizes: $<0.33$ (small), 0.33 to 0.55 (medium), and $>0.55$ (large). The correlation coefficient $\left(\mathrm{R}^{2}\right)$ was used to assess the goodness of fit for the statistical model and ranges from 0 to 1 , with 1 being a perfect fit. For large sample sizes, an $\mathrm{R}^{2}$ of 0.3 is considered acceptable.

\section{RESULTS}

\section{Patient Characteristics}

For the 2-year period of interest, 11,669 patients with a DTAA met our inclusion and exclusion criteria. Of these, $9106(78 \%)$ had undergone conventional OAR and 2563 $(22 \%)$ had undergone TEVAR.

The baseline characteristics were compared between the patients who had undergone OAR versus TEVAR (Table 1). The patients who had undergone TEVAR were older than those who had undergone OAR by almost 10 years $(P<.001)$ and had a significantly greater Deyo comorbidity score $(P<.001)$. In each group, the proportion of procedures was similar. The most common primary payer was Medicare for TEVAR patients $(66.9 \%)$ and private insurance for OAR patients $(49.4 \% ; P<.001)$. This discrepancy resulted from the age difference between the 2 groups. The patients in the highest income quartile constituted the largest proportion of patients who had undergone OAR (33.8\%) and the smallest proportion who had undergone TEVAR $(21.5 \%)$ compared with the other income quartiles. The opposite trend was noted for patients in the lowest income quartile. Most OARs (78.9\%) and TEVARs $(72.9 \%)$ were performed in hospitals with a large bed size (per the HCUP definition ${ }^{10}$ ). The proportion of white patients was greater in the OAR group than in the
TEVAR group $(88.7 \%$ vs $78.2 \% ; P<.001)$; the opposite was true for black patients $(4.7 \%$ vs $10.3 \% ; P<.001)$.

Although the prevalence of several comorbidities (Table 2) was significantly different between the 2 groups, the effect sizes were $<0.33$ for all differences. The prevalence of chronic peripheral vascular disease, previous cerebrovascular disease, chronic pulmonary disease, peptic ulcer disease, diabetes mellitus, and chronic kidney disease was greater in the TEVAR group than in the OAR group. However, a history of myocardial infarction and congestive heart failure was more common in the OAR group than in the TEVAR group.

\section{Mortality}

The unadjusted outcomes are summarized in Table 3 . The risk-adjusted outcomes are summarized in Table 4. The inhospital mortality rate was $2.3 \%$ for both groups. After adjustment for confounding factors and comorbidities, the type of surgical approach did not seem to affect the mortality risk. Increasing age correlated independently with a greater risk of mortality after surgical intervention, but gender and the comorbidity index did not (Table 4).

\section{Hospital LOS}

The mean unadjusted LOS was $8.8 \pm 7.9$ days for the OAR patients and $7.6 \pm 11.1$ days for the TEVAR patients $(P<.001)$. The median LOS was 7 days for the OAR patients (range 0-155 days) and 5 days for the TEVAR patients (range, 1-151 days). Multivariable linear regression analysis revealed that TEVAR was independently associated with a shorter hospital LOS (by 1.3 days; $P<.001$ ). Female gender was independently associated with a 1.5-day 
TABLE 2. Patient comorbidities

\begin{tabular}{|c|c|c|c|c|c|}
\hline Comorbidity & OAR $(n=9106)$ & TEVAR $(n=2563)$ & $\chi^{2}$ & $P$ value & $\varphi$ \\
\hline Chronic PVD & $368(4.0 \%)$ & $564(22.0 \%)$ & 878.398 & $<.001$ & 0.274 \\
\hline Previous MI & $10(0.1 \%)$ & $10(0.4 \%)$ & 9.189 & .005 & 0.028 \\
\hline Previous CHF & $1441(15.8 \%)$ & $264(10.3 \%)$ & 48.896 & $<.001$ & 0.065 \\
\hline Previous cerebrovascular disease & $278(3.1 \%)$ & $205(8.0 \%)$ & 123.315 & $<.001$ & 0.103 \\
\hline Chronic pulmonary disease & $1306(14.4 \%)$ & $881(34.4 \%)$ & 527.618 & $<.001$ & 0.213 \\
\hline Rheumatic disease & $110(1.2 \%)$ & $42(1.6 \%)$ & 2.888 & .09 & 0.016 \\
\hline Peptic ulcer disease & $25(0.3 \%)$ & $24(0.9 \%)$ & 20.957 & $<.001$ & 0.042 \\
\hline Mild liver disease & $40(0.4 \%)$ & $5(0.2 \%)$ & 3.104 & .1 & 0.016 \\
\hline Diabetes mellitus & $854(9.4 \%)$ & $352(13.7 \%)$ & 40.943 & $<.001$ & 0.059 \\
\hline Diabetic complications & $63(0.7 \%)$ & $25(1.0 \%)$ & 2.149 & .2 & 0.014 \\
\hline Hemiplegia or paraplegia & $61(0.7 \%)$ & $26(1.0 \%)$ & 3.209 & .09 & 0.017 \\
\hline Chronic kidney disease & $388(4.3 \%)$ & $313(12.2 \%)$ & 224.128 & $<.001$ & 0.139 \\
\hline HIV & $10(0.1 \%)$ & 0 & 2.817 & .1 & 0.016 \\
\hline Cancer & $120(1.3 \%)$ & $36(1.4 \%)$ & 0.116 & .7 & 0.003 \\
\hline Metastatic cancer & $18(0.2 \%)$ & $5(0.2 \%)$ & 0.001 & 1.0 & 0.001 \\
\hline
\end{tabular}

Data are presented as number (\%). OAR, Open aortic repair; TEVAR, thoracic endovascular aneurysm repair; $P V D$, peripheral vascular disease; $M I$, myocardial infarction; $C H F$, congestive heart failure; $H I V$, human immunodeficiency virus.

increase in the $\operatorname{LOS}(\mathrm{B}=1.53 ; 95 \%$ confidence interval $[\mathrm{CI}], 1.14-1.93 ; P<.001)$, and a unit increase in the Deyo comorbidity index score was associated with a 1-day increase in the hospital LOS (B = 1.09; 95\% CI, 0.65-1.52; $P<.001)$.

\section{Discharge Status}

The discharge was routine for $65.2 \%$ of the TEVAR patients but only $45.3 \%$ of the OAR patients $(P<.001)$, and TEVAR was independently associated with a 4 times greater likelihood of a routine discharge home (odds ratio, 4.76; 95\% CI, 3.48-4.63; $P<.001)$. In the multivariable regression model, age did not affect the discharge status, but female gender and greater comorbidity scores were associated with lower odds of a routine discharge (Table 4).

TABLE 3. Unadjusted outcomes of TEVAR versus OAR

\begin{tabular}{lcccc}
\hline \multicolumn{1}{c}{ Outcome } & $\begin{array}{c}\text { OAR } \\
(\mathbf{n}=\mathbf{9 1 0 6})\end{array}$ & $\begin{array}{c}\text { TEVAR } \\
(\mathbf{n}=\mathbf{2 5 6 3})\end{array}$ & $\begin{array}{c}\boldsymbol{P} \\
\text { value }\end{array}$ & $\begin{array}{c}\text { Effect } \\
\text { size }\end{array}$ \\
\hline Died during hospitalization & $209(2.3 \%)$ & $59(2.3 \%)$ & 1.0 & $0 *$ \\
Complications per patient & $0.51 \pm 0.79$ & $0.33 \pm 0.67$ & $<.001$ & $0.7 \dagger$ \\
Any complication & $3428(37.6 \%)$ & $588(22.9 \%)$ & $<.001$ & $0.128^{*}$ \\
Length of stay (d) & $8.77 \pm 7.9$ & $7.6 \pm 11.1$ & $<.001$ & $0.13 \dagger$ \\
Routine discharge & $4126(45.3 \%)$ & $1671(65.2 \%)$ & $<.001$ & $0.188^{*}$ \\
Intraoperative/procedure- & $2066(22.7 \%)$ & $294(11.5 \%)$ & $<.001$ & $0.116^{*}$ \\
$\quad$ related complications & & & & \\
Deep venous thrombosis & $38(0.4 \%)$ & $50(2.0 \%)$ & $<.001$ & $0.073^{*}$ \\
Infections & $488(5.4 \%)$ & $140(5.5 \%)$ & .8 & $0.002^{*}$ \\
Neurologic complications & $217(2.4 \%)$ & $64(2.5 \%)$ & .7 & $0.003^{*}$ \\
Pulmonary embolism & $32(0.4 \%)$ & $6(0.2 \%)$ & .4 & $-0.009^{*}$ \\
Respiratory complications & $951(10.4 \%)$ & $110(4.3 \%)$ & $<.001$ & $-0.089^{*}$ \\
Renal complications & $535(5.9 \%)$ & $157(6.1 \%)$ & .6 & $0.004^{*}$ \\
\hline
\end{tabular}

Data are presented as number (\%) or mean \pm standard deviation. OAR, Open aortic repair; TEVAR, thoracic endovascular aneurysm repair. $* \mathrm{Phi}(\varphi)$ was used to calculate effect size. $\nmid$ Cohen's d was used to calculate effect size.

\section{Overall Postoperative Complications}

Regarding postoperative complications, the total number of complications per patient was analyzed using linear regression analysis and adjusting for confounding variables. The risk of any complication was analyzed using logistic regression modeling. The linear regression analysis results indicated that TEVAR was associated with 1 fewer complication for every 3 patients $(\mathrm{B}=-0.334 ; P<.001)$, and TEVAR was associated with a lower risk of any complication (odds ratio, 0.39 ; 95\% CI, 0.26-0.58; $P<.001$ ). In a multivariable regression model, no baseline characteristic was associated with the risk of postoperative complications except for the Deyo comorbidity score, which approached statistical significance (odds ratio, $1.5 ; 95 \%$ CI, 0.99-2.57; $P=.056)$.

\section{Subgroup Analysis: Postoperative Complications}

In-hospital complications were segregated into different categories and analyzed further with logistic regression models (Table 5) to predict the independent effect of TEVAR versus OAR on each complication category. Regarding the specific categories of complications, TEVAR was independently associated with fewer intraoperative/ procedure-related, neurologic, and respiratory complications; however, the incidence of pulmonary embolism, renal complications, and infections did not differ between the 2 groups.

\section{Hospital Charges and Cost}

The unadjusted hospital charges were similar for the TEVAR and OAR patients $(\$ 116,329 \pm \$ 109,006$ vs $\$ 117,985 \pm \$ 80,823 ; P=.5)$. However, after risk adjustment using multivariable linear regression analysis, TEVAR was associated with an additional hospital charge of $\$ 6713(P<.01)$. Hospital charge information captured in 
TABLE 4. Independent predictors of outcomes

\begin{tabular}{|c|c|c|c|c|c|c|c|c|c|}
\hline \multirow[b]{2}{*}{ Predictor } & \multicolumn{3}{|c|}{ Death } & \multicolumn{3}{|c|}{ Routine discharge } & \multicolumn{3}{|c|}{ Any complication } \\
\hline & $P$ value & Odds ratio & $\mathbf{9 5} \% \mathrm{CI}$ & $P$ value & Odds ratio & $\mathbf{9 5} \% \mathrm{CI}$ & $P$ value & Odds ratio & $\mathbf{9 5} \%$ CI \\
\hline Age (y) & .01 & 1.079 & $1.017-1.145$ & .1 & 0.992 & $0.981-1.002$ & 1.0 & 1.001 & $0.963-1.040$ \\
\hline Female gender & .2 & 1.243 & $0.886-1.743$ & $<.001$ & 0.798 & $0.715-0.889$ & .9 & 1.025 & $0.740-1.420$ \\
\hline Deyo index & .2 & 0.669 & $0.377-1.188$ & $<.001$ & 0.784 & $0.695-0.885$ & .056 & 1.500 & $0.990-2.274$ \\
\hline TEVAR & .9 & 1.033 & $0.682-1.564$ & $<.001$ & 4.014 & $3.481-4.628$ & $<.001$ & 0.385 & $0.256-0.578$ \\
\hline
\end{tabular}

CI, Confidence interval; TEVAR, thoracic endovascular aneurysm repair.

the NIS represents the amount hospitals billed for services but does not reflect how much the hospital services actually cost or the specific amounts that the hospitals received in payment. The cost/charge ratios, formulated by the NIS for each hospital, were used to compute the estimated cost incurred by the hospital per case. The unadjusted hospital costs were lower for the TEVAR patients than for the OAR patients $(\$ 46,636 \pm \$ 46,303$ vs $\$ 48,974 \pm \$ 32,943$; $P<.05)$. However, multivariable linear regression analysis revealed that TEVAR was not independently associated with any cost benefit $(P=.2)$.

\section{DISCUSSION}

We analyzed the data from a large nationwide cohort of patients who had undergone TEVAR for isolated DTAA. Our results have provided detailed insight into the initial US implementation of TEVAR as a surgical modality. On average, the TEVAR patients were older than the OAR patients by almost 1 decade, and the TEVAR patients had more comorbidities. These differences occurred because, initially, TEVAR was primarily used for relatively highrisk patients who could not tolerate OAR, the standard treatment. Thus, OARs constituted $75 \%$ of the procedures for isolated DTAA. If the pattern of increasingly widespread use of endovascular repair for abdominal aortic aneurysms can be extrapolated to DTAA repair, a strong potential exists for increasing the proportion of TEVAR cases.

TABLE 5. Effect of TEVAR on outcomes after DTAA repair: Hierarchic multivariable regression models

\begin{tabular}{lcccc}
\hline \multicolumn{1}{c}{ Variable } & $\begin{array}{c}\boldsymbol{P} \\
\text { value }\end{array}$ & $\begin{array}{c}\text { Odds ratio or } \\
\mathbf{E x p}(\mathbf{B})\end{array}$ & $\mathbf{9 5 \%} \mathbf{C I}$ & $\mathbf{R}^{\mathbf{2}}$ \\
\hline Intraoperative complications & $<.001$ & 0.41 & $0.35-0.50$ & 0.14 \\
Infections & .3 & 0.88 & $0.68-1.14$ & 0.22 \\
Neurologic complications & $<.001$ & 0.16 & $0.09-0.29$ & 0.60 \\
Renal complications & .3 & 0.87 & $0.67-1.14$ & 0.26 \\
Respiratory complications & $<.001$ & 0.28 & $0.21-0.36$ & 0.18 \\
Pulmonary embolism & .5 & 1.51 & $0.51-4.47$ & 0.46 \\
Any complication & $<.001$ & 0.39 & $0.26-0.58$ & 0.93 \\
Total complications/patient & $<.001$ & $-0.33 *$ & -0.38 to -0.29 & 0.11 \\
Died during hospitalization & .9 & 1.03 & $0.68-1.56$ & 0.38 \\
Length of stay (d) & $<.001$ & $-1.27 *$ & -1.76 to -0.79 & 0.28 \\
Routine home discharge & $<.001$ & 4.01 & $3.48-4.63$ & 0.25 \\
\hline
\end{tabular}

*Multivariable linear regression used for continuous variable outcome measures. TEVAR, Thoracic endovascular aneurysm repair; DTAA, descending thoracic aortic aneurysm; $R^{2}$, correlation coefficient; other abbreviations as in Table 4.
The most common primary payer for TEVAR patients was Medicare, which correlated with these patients being older. A greater proportion of TEVAR patients than OAR patients were in the lowest quartile of income, and twice as many black patients had undergone TEVAR as had undergone OAR. One could speculate that these patients were more likely to have more comorbidities, which would have favored a less-invasive approach. In addition, because of the relative lack of data supporting the long-term reliability of TEVAR, OAR has been preferentially offered to younger patients. A significantly greater proportion of female patients underwent TEVAR, but female gender was not associated with greater mortality or morbidity risk in the regression models. Female gender, overall, was associated with lower odds of a routine discharge after intervention on the DTAA. A more detailed socioeconomic and demographic analysis is needed to understand the dynamics of this interaction. In addition, as clinicians gain experience with time, the proportion of TEVAR procedures performed in various socioeconomic, racial, and age groups is likely to change.

For older patients, the LOS must be considered in the context of the patient's discharge status. Shorter LOSs might not necessarily indicate better outcomes if the patients were discharged to nursing facilities or other healthcare venues. Because insurance companies have capped payments, hospitals have tended to aggressively incorporate social services early on in the care of these relatively complex patients to mobilize them fairly quickly to nursing facilities or arrange support services to shorten the hospital LOS. Our analysis showed that both the LOS and the proportion of nonroutine discharges were lower for TEVAR patients, suggesting a less morbid surgical procedure associated with quicker recovery.

Our analysis revealed that the mortality profiles for TEVAR and OAR were not different, even after risk adjustment. The overall unadjusted mortality rate was $2.3 \%$ for both groups. Although TEVAR was performed in patients with more comorbidities, a critical review of the regression model for mortality revealed that, surprisingly, the overall comorbidity score was not associated with mortality. In contrast, patient age had a significant independent effect on mortality. We believe that, as TEVAR becomes more widely adopted in the near future, the age difference between OAR and TEVAR patients is likely to disappear, 
which could potentially unmask a mortality benefit. Although a propensity-matching analysis would be ideal for answering this question, the sample weights and significant age discrepancy between the 2 groups prevented us from creating a large enough propensity-matched subsample of patients to perform an adequately powered analysis. As more data become available during the next few years, a propensity-matching analysis could become more feasible.

We found that TEVAR was independently associated with fewer procedure-related, neurologic, and respiratory complications than OAR, resulting in a $61 \%$ lower overall complication rate and, therefore, a greater proportion of routine discharges and shorter hospital LOSs. The lower incidence of neurologic and respiratory complications associated with TEVAR was not surprising, considering that the procedure involves no aortic cross-clamping, ischemic time, or thoracotomy. However, the benefits realized by the reduction in morbidity did not necessarily result in less mortality for the TEVAR patients.

The incidence of renal complications in the 2 groups was similar. The TEVAR patients were much older and received larger amounts of contrast agent, both during the actual procedure and during preoperative high-resolution computed tomographic angiography. However, patients who undergo OAR have transient renal ischemia because of aortic crossclamping, which is entirely avoided with TEVAR. We believe these risk factors, although unique to each group, have similar effects on the incidence of postoperative renal dysfunction. However, certain relevant data, such as the quantity of intravenous contrast used, whether patients underwent preprocedural renal prophylaxis, and the preoperative creatinine levels, were not available from the database we used.

We found that TEVAR was independently associated with a shorter hospital LOS by 1.3 days and a median hospital LOS of 5 days. As with any minimally invasive procedure, a major selling point would be the shorter LOS, which might result in lower hospital costs. However, the reduction in the LOS was only modest; thus, its financial benefits were less than what one might have anticipated. Although TEVAR only moderately reduced the hospital LOS, after adjustment for confounding factors, TEVAR was associated with a 4 times greater likelihood of a routine discharge to home than was OAR. Thus, the main benefit of TEVAR seems to be that most of the patients go home rather than to a skilled nursing or long-term healthcare facility. However, our discharge data did not include readmissions for complications or hospital visits for follow-up computed tomography. This was a limitation of our study because, compared with the complications of OAR, complications resulting from endovascular aortic stenting procedures are more likely to manifest after the patient has been discharged home.

Our study found that TEVAR was associated with significantly greater hospital charges. Stent grafts are much more expensive than the grafts traditionally used in OAR, and the overhead expenses of maintaining a hybrid suite to perform TEVAR are greater than those of a typical operating suite. This, and several other factors, have driven hospitals to charge more for TEVAR procedures.

Because the TEVAR patients stayed in the hospital for a shorter period, the overall cost to the hospital was approximately $\$ 2300$ less. However, the adjustment for confounding factors, including the LOS, cancelled this benefit, suggesting that the collateral/operating costs were similar between OAR and TEVAR. However, stent grafts are expensive, and as more graft modules are used, hospitals are more likely to lose revenue. ${ }^{17,18}$ Other expenses that would further reduce the cost benefit include the costs of additional graft modules to treat endoleaks and of followup computed tomography, which is routine after TEVAR. A more meaningful outcome variable would be hospital profits, but our database did not contain the information necessary to examine that variable.

A study by Orandi and colleagues ${ }^{19}$ was the first to examine the short-term (3-month) outcomes of TEVAR after the stent grafts were approved by the FDA. The initial FDA approval in 2005 allowed thoracic endovascular stent grafts to be marketed on a nationwide basis; however, by that time, a few centers had already been using TEVAR to treat aneurysms and other aortic disease for about 5 years. The familiarity with TEVAR at these centers allowed them to use this modality to treat thoracic aortic dissections, infections, rupture, and traumatic injuries and to perform staged procedures in patients with extensive aortic disease. Modifications of conventional principles and the use of adjunct procedures such as carotid subclavian bypass and transapical approaches ${ }^{20}$ have allowed surgeons to treat even more extensive thoracic aortic disease with endovascular approaches. In addition, the availability of TEVAR as a viable option has allowed surgeons to modify the open surgical approach to facilitate the treatment of aortic aneurysms in adjacent territories. For example, the use of the trifurcated graft technique to repair the aortic arch takes into consideration the need to create an adequate "landing zone" should the patient require TEVAR in the more distal aorta in the future.

In 2002, Najibi and colleagues ${ }^{21}$ reported the first comparison of TEVAR with open surgical repair in a series of 18 patients. The control groups included a historic cohort of patients who had undergone open repair during the previous 3 years. The short-term follow-up data showed that the endovascular group had significantly shorter operative times, shorter hospital and intensive care unit stays, and less operative blood loss. In 2005, Bavaria and colleagues ${ }^{22}$ reported the findings of the Phase II multicenter trial that compared the results of Gore TAG thoracic endograft placement $(\mathrm{n}=140)$ with those of traditional open repair $(\mathrm{n}=94)$. The cohort included a group of low-risk patients selected according to stringent inclusion and exclusion 
criteria. The short-term follow-up data revealed TEVAR to be associated with a lower incidence of spinal cord ischemia, fewer respiratory and renal complications, and a shorter hospital LOS. The pivotal results of the Evaluation of the Medtronic Vascular Talent Thoracic Stent Graft System for the Treatment of Thoracic Aortic Aneurysms (VALOR) trial supported the use of the Talent Thoracic Stent Graft System as a safe and effective alternative to open surgical repair in patients with DTAAs. These elderly patients, despite their significant comorbidities, had low mortality at 30 days, and TEVAR showed superior performance with respect to acute procedural outcomes and perioperative mortality. Although each study had different limitations and control group characteristics, the findings of our nationwide evaluation are consistent with these published results.

Our study was subject to the limitations inherent to its retrospective design and the nature of the database used. Our data represent a snapshot of real-world surgical practice in the first few years after FDA approval, during which the effect of the learning curve on TEVAR could have been substantial but would have been difficult to quantify. The data set included all TEVAR and OAR patients in the United States but only for a 2-year period. This sample, although the largest to date, was not large enough to determine the specific effects of hospital characteristics (eg, whether or not the hospital was a dedicated aortic center) on the outcomes of TEVAR. However, making this determination will become more feasible as data from more recent years become available.

The study's retrospective design imposed selection biases in terms of whether OAR or TEVAR was chosen for a given patient. That said, database studies are typically limited by the contents of the database, which might not include data regarding every potentially relevant variable. For example, the NIS database does not include any information regarding aneurysmal extent, size, or symptoms or the functional status of the patient. Also, neurologic complications had to be considered as a single outcome because no specific ICD-9-CM codes are available to differentiate between postoperative paraplegia and stroke. In addition, only data regarding the total LOS, reflecting the period from preoperative admission to discharge, were available. Although the postoperative LOS would be more useful for identifying benefits specific to the procedure itself, this information was not available.

Because of the administrative nature of the database, reporting bias could have been present owing to coding errors. Statistically, such a bias should have affected the 2 groups of patients equally. Also, hard end points such as mortality, LOS, and hospitalization costs are less likely to be affected by coding errors than are more subjective clinical end points. Long-term follow-up data to capture future reinterventions are not available from the NIS database. Similarly, as reported in published studies, most failures of TEVAR occur after discharge, rather than during the index hospitalization. ${ }^{23}$ Therefore, because of the lack of follow-up or readmission data in the NIS database, our conclusions regarding the in-hospital outcomes of TEVAR should not be extrapolated to the short- or long-term outcomes. Despite these limitations, the NIS database provided the best overall sample for a nationwide assessment, because it included data from a large, varied group of US hospitals.

\section{CONCLUSIONS}

Our nationwide study has shown that TEVAR resulted in outcomes that were comparable, if not superior, to those of OAR for isolated DTAA, even though TEVAR was selectively performed in patients who were almost 1 decade older than the OAR patients. Although a shorter hospital LOS and greater odds of routine home discharge favored TEVAR, the in-hospital mortality profiles of TEVAR and OAR were similar. Likewise, TEVAR was not associated with any risk-adjusted cost benefit compared with OAR, although the effects of race, gender, and socioeconomic status on the clinical value of TEVAR require additional study. The primary benefit in the risk-adjusted morbidity profile for TEVAR stemmed from the lower incidence of neurologic, respiratory, and intraoperative complications. Follow-up studies involving more extensive databases that capture the readmission data, interventions, follow-up imaging findings, and pertinent costs would be more informative about the long-term effectiveness of TEVAR. Although our findings should not be used to dictate changes in clinical practice, the results have provided unique insights from a nationwide perspective. This distinguishes them from the findings of single-institution studies, which have probably been biased by local expertise and institutional culture.

The authors thank Stephen N. Palmer, PhD, ELS, for editorial assistance.

\section{References}

1. DeBakey ME, Cooley DA. Successful resection of aneurysm of thoracic aorta and replacement by graft. JAMA. 1953;152:673-6.

2. Parodi JC, La Mura R. Endovascular aortic devices: The Parodi and Palmaz system. Surg Technol Int. 1996;5:290-6.

3. Parodi JC, Palmaz JC, Barone HD. Transfemoral intraluminal graft implantation for abdominal aortic aneurysms. Ann Vasc Surg. 1991;5:491-9.

4. Dake MD, Miller DC, Semba CP, Mitchell RS, Walker PJ, Liddell RP. Transluminal placement of endovascular stent-grafts for the treatment of descending thoracic aortic aneurysms. N Engl J Med. 1994;331:1729-34.

5. Makaroun MS, Dillavou ED, Kee ST, Sicard G, Chaikof E, Bavaria J, et al. Endovascular treatment of thoracic aortic aneurysms: Results of the phase II multicenter trial of the GORE TAG thoracic endoprosthesis. J Vasc Surg. 2005;41:1-9.

6. Adams JD, Angle JF, Matsumoto AH, Peeler BB, Arslan B, Cherry KJ, et al. Endovascular repair of the thoracic aorta in the post-FDA approval era. $J$ Thorac Cardiovasc Surg. 2009; 137:117-23.

7. Fernandez V, Mestres G, Maeso J, Dominguez JM, Aloy MC, Matas M. Endovascular treatment of traumatic thoracic aortic injuries: Short- and medium-term follow-up. Ann Vasc Surg. 2010;24:160-6.

8. McPhee JT, Asham EH, Rohrer MJ, Singh MJ, Wong G, Vorhies RW, et al. The midterm results of stent graft treatment of thoracic aortic injuries. J Surg Res. 2007;138:181-8. 
9. Lee AM, Melby SJ, Damiano RJ Jr. The surgical treatment of atrial fibrillation. Surg Clin North Am. 2009;89:1001-20, x-xi.

10. Healthcare Cost Utilization Project, Nationwide Inpatient Sample (NIS). Rockville, MD: Agency for Healthcare Research and Quality; 2001.

11. International classification of diseases, 9 th revision, clinical modification. 5 th ed. Washington, DC: US Department of Health and Human Services, Public Health Service; 1988 .

12. Deyo RA, Cherkin DC, Ciol MA. Adapting a clinical comorbidity index for use with ICD-9-CM administrative databases. J Clin Epidemiol. 1992;45: 613-9.

13. Gopaldas RR, Chu D, Dao TK, Huh J, LeMaire SA, Coselli JS, et al. Predictors of surgical mortality and discharge status after coronary artery bypass grafting in patients 80 years and older. Am J Surg. 2009;198:633-8.

14. LeMaire A, Cook C, Tackett S, Mendes DM, Shortell CK. The impact of race and insurance type on the outcome of endovascular abdominal aortic aneurysm (AAA) repair. J Vasc Surg. 2008;47:1172-80.

15. Cohen J. Statistical power analysis for the behavioral sciences. 2nd ed. Hillsdale, NJ: L. Erlbaum Associates; 1988.

16. Lipsey MW. Design sensitivity: statistical power for experimental research. Newbury Park, Calif: Sage Publications; 1990.

17. Birch SE, Stary DR, Scott AR. Cost of endovascular versus open surgical repair of abdominal aortic aneurysms. Aust N Z J Surg. 2000;70:660-6.

18. Murphy EH, Beck AW, Clagett GP, DiMaio JM, Jessen ME, Arko FR. Combined aortic debranching and thoracic endovascular aneurysm repair (TEVAR) effective but at a cost. Arch Surg. 2009;144:222-7.

19. Orandi BJ, Dimick JB, Deeb GM, Patel HJ, Upchurch GR Jr. A population-based analysis of endovascular versus open thoracic aortic aneurysm repair. $J$ Vasc Surg. 2009;49:1112-6.

20. Szeto WY, Moser WG, Desai ND, Milewski RK, Cheung AT, Pochettino A, et al. Transapical deployment of endovascular thoracic aortic stent graft for an ascending aortic pseudoaneurysm. Ann Thorac Surg. 2010;89:616-8.

21. Najibi S, Terramani TT, Weiss VJ, MacDonald MJ, Lin PH, Redd DC, et al. Endoluminal versus open treatment of descending thoracic aortic aneurysms. $J$ Vasc Surg. 2002;36:732-7.

22. Bavaria JE, Appoo JJ, Makaroun MS, Verter J, Yu ZF, Mitchell RS. Endovascular stent grafting versus open surgical repair of descending thoracic aortic aneurysms in low-risk patients: A multicenter comparative trial. J Thorac Cardiovasc Surg. 2007;133:369-77.

23. Neuhauser B, Greiner A, Jaschke W, Chemelli A, Fraedrich G. Serious complications following endovascular thoracic aortic stent-graft repair for type B dissection. Eur J Cardiothorac Surg. 2008;33:58-63.

\section{Discussion}

Dr John A. Kern (Charlottesville, Va). Thank you. I have nothing to disclose.

Dr Gopaldas and his colleagues from Houston have presented to us the largest review to date of thoracic endovascular aneurysm repair (TEVAR) versus open repair for isolated descending thoracic aortic aneurysms. This is a large database, and the results were more or less as expected. These particular results square up nicely with previously published reports, as you have pointed out, and, in general, the lower surgical complications and lower neurologic and respiratory complications should be looked on favorably from a healthcare economics standpoint. Interestingly, however, although the complications were lower in the TEVAR patients, the procedure-related hospital stay and mortality was actually identical between the 2 groups. This is perhaps a little unexpected given the lower complications in the TEVAR group.

I would like to emphasize the study for what it is and what it is not, and then I have 3 questions. First, the sheer number of patients analyzed makes this study noteworthy, sample size is not an issue, and TEVAR was proven safe and, indeed, perhaps superior to open repair. In addition, this study represents a true broad snapshot, if you will, of the real-world experience with the early widespread adoption of what was at the time a new Food and Drug Adminis- tration-approved technology, TEVAR, and it showed that it stood up nicely to the reference standard, open repair. This is particularly impressive when realizing that data was collected from more than 1000 hospitals nationwide, as you pointed out. Therefore, perhaps the results are not too terribly skewed by the high-volume, experienced academic referral centers where stent grafting technology was developed, refined, and validated by the experts. Indeed, perhaps some lower volume centers were included in this trial and their data were analyzed, and this is good.

What is this study not? First, this study was not a prospective randomized trial, and this study did not use propensity matching to compare similar patients with subsequent outcomes. This study did use an administrative database, which was probably very barebones and, as you said, likely subject to coding errors and biases. Although some patient demographics were collected-for example, we saw that the TEVAR patients were older and perhaps a bit sicker-we have no information concerning the actual nature, pathology, location, extent, or size of the aneurysms treated. Thus, it is possible that you were comparing apples and oranges. Second, no information is known about the downstream subsequent consequences after either TEVAR or open repair. This in fact is a particularly important feature of stent grafting, even more so now as we tackle more complex and challenging aortic pathologic findings in which type I endoleaks can be seen in as many as $20 \%$ of patients. These situations obviously require significant downstream resource use and expertise. The need for frequent reimaging and reinterventions could make TEVAR a bit less attractive in the long run for both patients and third-party payers. Although TEVAR might appear favorable compared with open repair during the initial hospitalization, the cumulative cost and consequences of subsequent hospitalizations and interventions are still unknown. So I have 3 questions.

First, what do you hypothesize was the major mode of death in the TEVAR group? Complications, specifically life-altering respiratory and neurologic complications, appeared less frequently in TEVAR, yet the mortality was similar. Is it possible the increased mortality was related to the vascular access complications associated with the early adoption of TEVAR, and do you think these vascular complications and mortality have improved in the 3 years?

Dr Gopaldas. Dr Kern, thank you for your very detailed perusal of our manuscript and for your extremely valuable comments. My first thing in response to your comment that we do not have information on the follow-up, which is certainly a very valid point, one thing that we do have to point out is that the routine discharge rates were greater with TEVAR. Thus, an older patient was more likely to go home, and, yes, come back for a reintervention, but then a patient of a similar age who had undergone open repair was probably going to end up in a nursing home and stay there for a long time and probably not go home. So, the critical issue here is we do not have information on either of these, whether they go to a nursing home, how much it costs, or if they come back for reinterventions for TEVAR procedures. That is certainly a limitation we acknowledge.

In answer to your question about the mortality difference that was not seen, which was actually interesting considering that TEVAR independently predicted lower complications. To address this, we analyzed our groups separately, TEVAR patients and open 
aortic repair. We conducted a regression model to determine what actually were the predictors of mortality within these groups. Also, I am going to point out 2 factors: one is intraoperative complications, the other is respiratory complications.

Now, TEVAR independently predicted a lower incidence of intraoperative complications; however, in the regression model separately, when an intraoperative complication occurred in the open aortic repair group, it did not independently translate to mortality. The odds ratio was 1.3 , but the $P$ value was not significant. In contrast, in a TEVAR patient, if they developed an intraoperative complication, the $P$ value was significant and the odds ratio was about 4.5. So what this tells us is TEVAR does decrease the incidence of intraoperative complications, but when an intraoperative complication does occur in a TEVAR patient, it is more likely to result in a grave consequence, such as death.

The same issue occurred with renal complications, because TEVAR did not translate to any difference in renal complications, but when we separately analyzed the 2 groups, a renal complication in an open aortic repair was associated with a 5 times risk of mortality, but in a TEVAR patient, a renal complication was associated with a 12 times risk of mortality.

So what I think we are seeing here are 2 things: one is the effect of TEVAR on the incidence of these complications and the second is the failure to rescue. Also, I think when TEVAR patients develop a complication, the failure to rescue from these complications by either the surgical team or the hospital was higher, and, as a result, they faced a greater mortality. I believe this is what seems to compensate and balance out the advantages of TEVAR and thus contributing to no overall difference.

To look at the other aspect, the length of stay was not different. We do not have information on the postoperative length of stay. So that is another limitation.

Dr Kern. That is a good analysis of those results.

Do you have any information from this database of the results as they compare to high-volume or low-volume centers? These data were captured when pretty straightforward aneurysms were being treated. You can see how lower-volume centers can treat that and do it well. We are starting to treat very complex aortic pathologic features. So, based on whether you know the results of low- versus high-volume centers, when it comes to very difficult dissections and disorders like that, should those still be treated by everyone?
Dr Gopaldas. That is certainly a very valid question. One of the factors we included in our analysis was hospital size, and that did not seem to affect the outcome of these procedures, although I must say this is an isolated descending thoracic aneurysm, which is fairly straightforward. Also, these cases, if a community hospital can do it well, it probably should be done there, rather than just being referred to an aortic center. The primary care physician is probably going to find a cardiologist or a vascular surgeon who can just push a guide wire up higher into the arch and deploy these stents, and these patients are not going to be knocking on the door of cardiac surgeons. But then if you are looking at complex aortic procedures, such as an arch in which extensive debranching is required or a thoracoabdominal aneurysm, that is a separate issue. For those patients, data are already available that have supported that performing these complex procedures in high-volume centers is justified. So for a straightforward isolated descending, I do not think these patients need to be shunted to an aortic center per se, but when it is complex requiring some debranching, absolutely, I think.

Dr Hanni Shennib (Phoenix, Ariz). I enjoyed your presentation very much, and it actually confirms a lot of the other meta-analyses that were done more recently, including a publication in the American Journal of Cardiology. The question I have to you is a follow-up on your comment regarding intraoperative complications and that the risk and complication rate, including risk and mortality, and what you quoted as rescue issues seems to be higher in the TEVAR group than in the open group. Can you perhaps retrieve information regarding who is billing for the procedure, whether it is co-billed between a cardiologist or an interventionalist and a surgeon versus the whole operation being done by an endovascular surgeon and perhaps come to some findings in regard to the failure to rescue? How many of those procedures, the TEVAR procedures, are done primarily by the endovascular service (i.e., billed by the endovascular service) versus billed by the cardiologist or interventional radiologist in the presence of a surgeon?

Dr Gopaldas. That information, unfortunately, is not available in this database. The Medicare database captures that in more detail, but this database does have that limitation. It does not differentiate between who is doing it. Whether a vascular surgeon or an interventionalist or a cardiologist or a cardiac surgeon is doing the procedure cannot be addressed with this database. 
APPENDIX 1. International Classification of Diseases, Ninth Revision, Clinical Modification codes used for inclusion/exclusion of study group patients

\begin{tabular}{|c|c|}
\hline \multicolumn{2}{|c|}{ Codes used for inclusion } \\
\hline 441.2 & $\begin{array}{l}\text { Diagnosis code: Thoracic aneurysm without mention } \\
\text { of rupture }\end{array}$ \\
\hline 39.73 & $\begin{array}{l}\text { Procedure code: Endovascular implantation of graft } \\
\text { in thoracic aorta }\end{array}$ \\
\hline 38.45 & $\begin{array}{l}\text { Procedure code: Resection of vessel with replacement; } \\
\text { thoracic aorta }\end{array}$ \\
\hline \multicolumn{2}{|c|}{ Diagnosis codes used for exclusion } \\
\hline 441.1 & Thoracic aneurysm, ruptured \\
\hline 441.3 & Abdominal aneurysm, ruptured \\
\hline 441.4 & Abdominal aneurysm without mention of rupture \\
\hline 441.5 & Aortic aneurysm of unspecified site, ruptured \\
\hline 441.6 & Thoracoabdominal aneurysm, ruptured \\
\hline 441.7 & $\begin{array}{l}\text { Thoracoabdominal aneurysm, without mention of } \\
\text { rupture }\end{array}$ \\
\hline 441.9 & $\begin{array}{l}\text { Aortic aneurysm of unspecified site without mention } \\
\text { of rupture }\end{array}$ \\
\hline 441.00 & Aortic dissection, unspecified site \\
\hline 441.01 & Aortic dissection, thoracic \\
\hline 441.02 & Aortic dissection, abdominal \\
\hline 441.03 & Aortic dissection, thoracoabdominal \\
\hline 446.0 & Polyarteritis nodosa \\
\hline 446.1 & Kawasaki disease \\
\hline 446.2 & Hypersensitivity angiitis \\
\hline 446.3 & Lethal midline granuloma \\
\hline 446.4 & Wegener's granulomatosis \\
\hline 446.5 & Giant cell arteritis \\
\hline 446.6 & Thrombotic microangiopathy \\
\hline 446.7 & Takayasu disease; aortic arch arteritis \\
\hline 758.6 & Turner syndrome; gonadal dysgenesis \\
\hline 759.82 & Marfan syndrome \\
\hline \multicolumn{2}{|c|}{ Procedure codes used for exclusion } \\
\hline $39.73+38.45$ & $\begin{array}{l}\text { Indicates conversion during, or reintervention after, } \\
\text { thoracic aneurysm repair }\end{array}$ \\
\hline 39.71 & Endovascular implantation of graft in abdominal aorta \\
\hline 38.44 & Resection of vessel with replacement; abdominal aorta \\
\hline
\end{tabular}

Thoracoabdominal aneurysm, ruptured
APPENDIX 2. International Classification of Diseases, Ninth Revision, Clinical Modification codes used to identify procedure-related complications

\begin{tabular}{ll}
\hline 423.0 & Hemopericardium \\
997.1 & Cardiac arrest or cardiorespiratory failure \\
996.0 & Mechanical complication due to an implant/graft \\
996.74 & Thrombosis, fibrosis, hemorrhage, embolism due to \\
& $\quad$ vascular graft \\
998.31 & Disruption of surgical wound \\
997.71 & Vascular complications of mesenteric artery \\
998.0 & Postoperative shock \\
998.11 & Hemorrhage \\
998.12 & Hematoma \\
998.13 & Seroma \\
E878.2 & Surgical procedure using prosthetic vascular graft directly \\
& $\quad$ causing abnormal patient reaction/complication \\
\hline
\end{tabular}

